\title{
Opportunities and challenges in synthetic oligosaccharide and glycoconjugate research
}

\author{
Thomas J. Boltje, Therese Buskas and Geert-Jan Boons ${ }^{\star}$
}

\begin{abstract}
Synthetic oligosaccharides and glycoconjugates are increasingly used as probes for biological research and as lead compounds for drug and vaccine discovery. These endeavours are, however, complicated by a lack of general methods for the routine preparation of these important compounds. Recent developments such as one-pot multistep protecting-group manipulations, the use of unified monosaccharide building blocks, the introduction of stereoselective glycosylation protocols, and convergent strategies for oligosaccharide assembly, are beginning to address these problems. Furthermore, oligosaccharide synthesis can be facilitated by chemo-enzymatic methods, which employ a range of glycosyl transferases to modify a synthetic oligosaccharide precursor. Glycosynthases, which are mutant glycosidases, that can readily form glycosidic linkages are addressing a lack of a wide range of glycosyltransferases. The power of carbohydrate chemistry is highlighted by an ability to synthesize glycoproteins.
\end{abstract}

T here is a growing appreciation that post-translational modifications, such as glycosylation, dramatically increase protein complexity and function ${ }^{1-6}$. For example, almost all cell surface and secreted proteins are modified by covalently linked carbohydrate moieties, and the glycan structures on these glycoproteins have been implicated as essential mediators in processes such as protein folding, cell signalling, fertilization, embryogenesis, neuronal development, hormone activity and the proliferation of cells and their organization into specific tissues. In addition, overwhelming data supports the relevance of glycans in pathogen recognition, inflammation, innate immune responses and the development of autoimmune diseases and cancer ${ }^{7-10}$. The importance of protein glycosylation is also underscored by the developmental abnormalities observed in a growing number of human disorders known as congenital disorders of glycosylation, caused by defects in the glycosylation machinery ${ }^{11}$.

Polysaccharides are major constituents of the microbial cell surfaces and, for example, the bacterial cell wall can contain relatively large amounts of capsular polysaccharides (CPS) or lipopolysaccharides (LPS $)^{12}$. These components are important virulence factors in that they promote bacterial colonization, block phagocytosis and interfere with leukocyte migration and adhesion. CPS and LPS can be recognized by receptors of the innate immune system leading to the production of cytokines, chemokines and cellular adhesion molecules ${ }^{13-16}$. With a few exceptions, bacterial polysaccharides can induce an adaptive immune response and, not surprisingly, bacterial saccharides have been employed for the development of vaccines for several pathogens ${ }^{17-20}$.

\section{Synthetic oligosaccharides for glycobiology}

A major obstacle in glycobiology and glycomedicine is the lack of pure and structurally well-defined carbohydrates and glycoconjugates $^{21}$. These compounds are often found in low concentrations and in microheterogeneous forms, greatly complicating their isolation and characterization. In many cases, well-defined oligosaccharides can only be obtained by chemical or enzymatic synthesis ${ }^{22-28}$ and such compounds are increasingly used to address important problems in glycobiology research and for vaccine and drug discovery. For example, the Consortium of Functional Glycomics (CFG) has employed a chemoenzymatic approach for the preparation of a library of over 400 oligosaccharides derived from $\mathrm{N}$ - and $\mathrm{O}$-linked glycoproteins and glycolipids ${ }^{29,30}$. These compounds, which are equipped with an artificial aminopropyl spacer, have been covalently attached to $N$-hydroxysuccinimide-activated glass slides and the resulting microrray has found wide use for profiling the specificity of a diverse range of glycan-binding proteins, such as C-type lectins, siglecs, galectins and anticarbohydrate antibodies. Glycan array technology has also been employed for rapid assessment of influenzae virus receptor specificity due to the species-specific nature of the interaction between virus and host glycans ${ }^{31}$.

Synthetic heparin and heparan sulfate fragments. The power of organic carbohydrate synthesis has also been demonstrated by the development of a fully synthetic heparin fragment used for the treatment of deep vein thrombosis in humans ${ }^{32-34}$. Heparin and heparan sulfate are naturally occurring polydisperse linear polysaccharides that are heavily $\mathrm{O}$ - and $\mathrm{N}$-sulfated ${ }^{5}$. For more than eighty years, heparin isolated from porcine mucosal tissue has been used as an anticoagulant drug. It exerts its activity by binding to the plasma protein antithrombin III (ATIII) causing a conformational change that results in its activation through an increase in the flexibility of its reactive site loop. The activated ATIII then inactivates thrombin and other proteases involved in blood clotting, most notably factor $\mathrm{Xa}$. The ATIII-binding region of heparin consists of a unique pentasaccharide domain. A fully synthetic analogue (Arixtra, Fig. 1a) of this domain has been developed, which is being produced on a multikilogram scale for the treatment of deep vein thrombosis ${ }^{32,33}$. Unlike heparin, the synthetic derivative is easy to characterize and has a much-improved subcutaneous bioavailability.

Several other synthetic heparin fragments are being developed for anticoagulation therapy, and the most exciting example is Idraparinux (Fig. 1b) ${ }^{32}$. This compound, which contains methyl ethers instead of hydroxyls and $\mathrm{O}$-sulfates instead of $\mathrm{N}$-sulfates, is much easier to synthesize than Arixtra, has an increased half-life and a much improved potency. The latter property seems to be due to the presence of the methyl ethers, which induce the biologically active ${ }^{2} \mathrm{~S}_{0}$ conformation of the iduronic acid moiety.

The importance of synthetic heparin for anticoagulation therapy was recently highlighted by the discovery of batches of heparin that were associated with anaphylactoid-type reactions, leading to hypotension and which resulted in nearly 100 deaths ${ }^{35,36}$. These adverse reactions were traced to contamination with a 

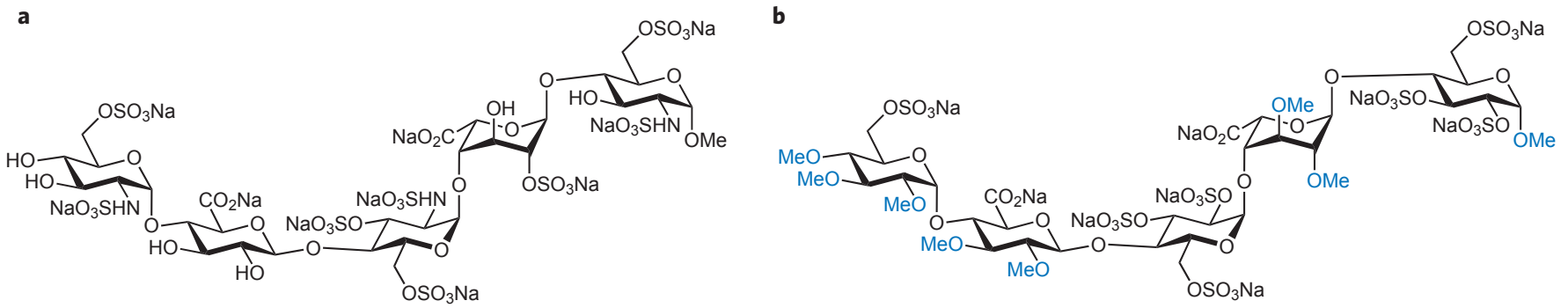

c

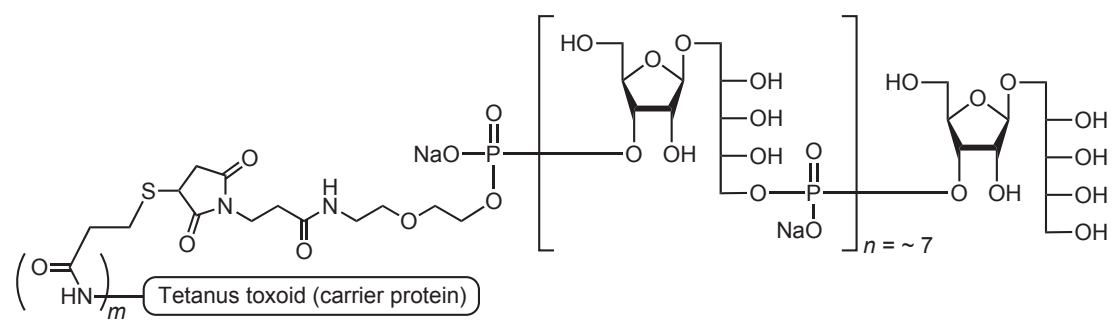

d

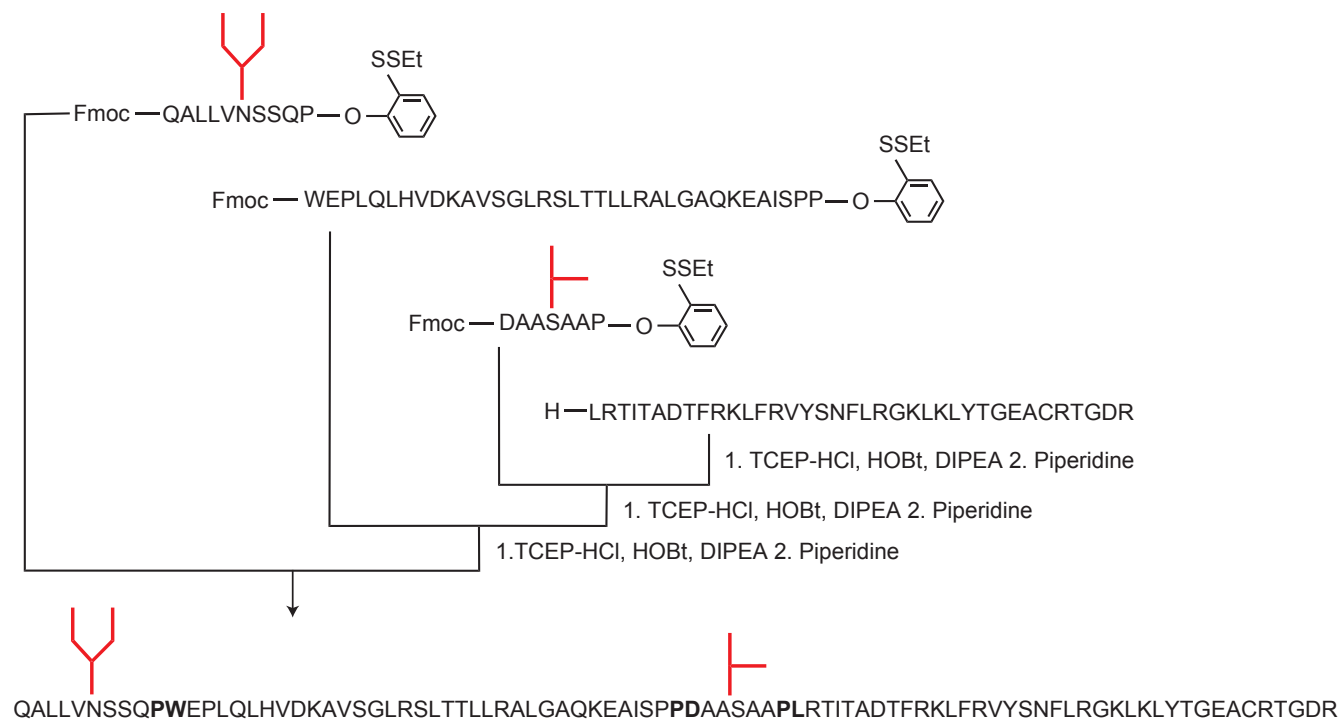

Figure 1 | Examples of biologically active synthetic oligosaccharide and glycopeptide constructs. a, Structure of the anticoagulant drug Arixtra. b, Structure of the promising anticoagulant candidate drug Idraparinux, in which the methyl ethers are highlighted in blue. c, Synthetic conjugate polysaccharide vaccine against Haemophilus influenzae type b. d, Synthesis of the erythropoietin (EPO; from amino acids 78-166) glycopeptide through sequential fragment condensation using auxiliary-based cysteine-free native chemical ligation. Glycans are shown in red and the ligation sites are shown in bold, amino acids are represented by their single-letter codes. DIPEA, N,N-diisopropylethylamine; HOBt, 1-hydroxybenzotriazole; TCEP-HCl, tris(2-carboxyethyl)phosphine hydrochloride.

semi-synthetic over-sulfated chondroitin sulfate, which is a popular shellfish-derived oral supplement for arthritis.

There is a growing body of literature indicating that glycosylaminoglycans (GAGs), such as heparin and heparan sulfate, can have profound physiological effects on lipid transport and adsorption, cell growth and migration and development ${ }^{5}$. Alterations in GAG expression has been associated with cancer and, for example, significant changes in the structure of GAGs has been reported in the stroma surrounding tumours, which is important for tumour growth and invasion. GAGs also have important neurobiological functions and examples include neuroepithelial growth and differentiation, neurite outgrowth, nerve regeneration, axonal guidance and branching, deposition of amyloidotic plaques in Alzheimer's disease, and astrocyte proliferation ${ }^{37}$. It is to be expected that synthetic analogues of heparin may find application in the treatment of several neurodiseases, cancer and infection ${ }^{33,34,37-42}$.

Carbohydrate-based prophylactic and therapeutic vaccines. Synthetic oligosaccharide epitopes offer promising possibilities for the development of vaccines for the prevention of infectious diseases such as Haemophilus influenzae type b, HIV, Plasmodium falciparum, Vibrio cholerae, Cryptococcus neoformans, Streptococcus pneumoniae, Shiga toxin, Neisseria meningitides, Bacillus anthracis and Candida albicans $s^{17,18,43,44}$. Natural polysaccharides conjugated to carrier proteins have been successfully developed as human vaccines, however, their use is associated with problems such as the destruction of vital immuno-dominant features during the chemical conjugation to a carrier protein ${ }^{17}$. Furthermore, 
natural polysaccharides show significant heterogeneity, which may compromise the reproducibility of their production and can contain highly toxic components that may be difficult to remove.

Fortunately, organic synthesis can provide carbohydrate epitopes in high purity and in relatively large amounts for controlled conjugation to a carrier protein. In such an approach, synthetic saccharides are equipped with an artificial spacer to facilitate selective conjugation to a carrier protein. In general, antibodies recognize epitopes that are no larger than a hexasaccharide. Although challenging, oligosaccharides of this length can be obtained by organic synthesis. The recent approval of a human vaccine based on a synthetic carbohydrate illustrates the potential use of organic synthesis to develop glycoconjugate vaccines $s^{45}$. The vaccine prevents infection by Haemophilus influenzae type b, a bacterium that causes pneumonia and meningitis in infants and young children. The carbohydrate epitope of this vaccine is an oligo-ribosylribitol phosphate, which was synthesized by a one-pot solution-phase oligomerization process (Fig. 1c). The synthetic compound is equipped with an artificial spacer that contains a maleimide moiety. The latter functionality allowed a controlled coupling of the synthetic oligosaccharide with a carrier protein that was modified by thiol moieties.

Synthetic oligosaccharides have also been employed for the development of therapeutic vaccines for cancer. The over-expression of oligosaccharides such as Globo- $\mathrm{H}$, Lewis ${ }^{\mathrm{Y}}$ and $\mathrm{Tn}$ antigen is a common feature of oncogenic transformed cells, and a broad and expanding body of preclinical and clinical studies demonstrates ${ }^{46-48}$ that naturally acquired, passively administered, or actively induced antibodies against carbohydrate-associated tumour antigens are able to eliminate circulating tumour cells and micro-metastases in cancer patients. The development of tumour-associated saccharides as cancer vaccines has been complicated because they are self-antigens and consequently tolerated by the immune system. The shedding of antigens by the growing tumour reinforces this tolerance. In addition, foreign carrier proteins, such as KLH and BSA, and the linker that attaches the saccharides to the carrier protein can elicit strong B-cell responses, which may lead to the suppression of antibody responses against the carbohydrate epitope. These problems are being addressed by the design, chemical synthesis, and immunological evaluation of a number of fully synthetic vaccine candidates that have the potential to overcome the poor immunogenicity of tumour-associated carbohydrates and glycopeptides ${ }^{49}$.

Glycopeptide and glycoprotein therapeutics. Synthetic oligosaccharides are also being employed for the preparation of well-defined glycoproteins ${ }^{50}$. Protein-based drugs represent approximately a quarter of new drug approvals with a majority being glycoproteins. The carbohydrate moiety of a glycoprotein is important for its pharmacokinetic properties, is involved in tissue targeting, and can modulate biological activities. Therefore, it is critical to control the exact chemical composition of the oligosaccharide moieties for the development of glycoprotein therapeutics. However, protein glycosylation is not under direct genetic control, and results in the formation of a heterogeneous range of glycoforms that possess the same peptide backbone but differ in the nature and site of glycosylation. Several strategies are being pursued to prepare well-defined mammalian glycoforms of glycoproteins, which include enzymatic remodelling of the oligosaccharide portion of glycoproteins, genetic engineering of the expression host, and the use of an orthogonal synthetase-tRNA pair that genetically encodes a glycosylated amino acid in response to a stop codon (an Amber codon) ${ }^{51}$.

A combination of site-directed mutagenesis and chemical attachment of synthetic oligosaccharides offers another unique approach for the preparation of well-defined glycoprotein $s^{52}$. This strategy involves the introduction of a cysteine in a protein, which can be site-selectively coupled with a synthetic saccharide containing an electrophilic moiety such as glycosyl iodoacetamides, thiols, dithiopyridines and methanethiosulfonates to give a well-defined neoglycoprotein. Recently, it has been demonstrated that the unnatural amino acid azido-homoalanine (Aha) can be incorporated into proteins by employing a Met(-)auxotropic strain, Escherichia coli B834(DE3), that is fed the unnatural amino acid instead of methionine $^{53}$. A subsequent click reaction between an alkyne-containing glycoside and the azide-modified protein gave triazole-linked glycoproteins in good yields.

The advent of native chemical ligation combined with the ability to prepare complex glycans has made it possible to synthesize glycoproteins. For example, the Danishefsky group has developed a strategy entailing the chemical synthesis of complex carbohydrates in their hemiacetal form, followed by Kochetkov amination, Lansbury amidation of a peptide, followed by auxiliary-based cysteine-free ligation to merge several glycopeptide and peptide fragments (Fig. 1d $)^{54}$. The approach was applied to the chemical synthesis of glycoproteins that carry multiple $\mathrm{N}$ - and $\mathrm{O}$-linked glycans such as $\beta$-human follicle-stimulating hormone ( $\beta$-hFSH), prostate-specific antigen (PSA) and part of human erythropoietin (EPO; from amino acids 78-166). These impressive syntheses showcase the power of chemical synthesis of carbohydrates and glycopeptides and the impact it can have on large-molecule 'biologicals'.

Multivalent glycomaterials. The ability to synthesize complex oligosaccharides is offering exciting opportunities for preparing glycopolymers, glycodendrimers and glyconanoparticles ${ }^{55-58}$. These materials are receiving increasing attention because monovalent saccharides often have weak interactions with their protein receptors. However, in nature, carbohydrate-binding proteins often exist as higher-order oligomeric structures presenting multiple binding sites, acting as 'polydentate' donors, thereby circumventing the intrinsic weak binding interactions of monovalent ligands ${ }^{59,60}$. Furthermore, cell-surface glycoproteins can be organized in domains by multivalent interactions with soluble lectins. The formation of lectin-saccharide lattices on the cell surface can thus organize the plasma membrane into specialized domains that perform unique functions ${ }^{61}$. For example, it has been found that Galectin-1, which is a lactoside-binding lectin, can selectively form complexes containing either the cell surface proteins CD45 and $\mathrm{CD} 3$ or $\mathrm{CD} 7$ and $\mathrm{CD} 43$, resulting in segregation of $\mathrm{CD} 3 / \mathrm{CD} 45$ from $\mathrm{CD} 7 / \mathrm{CD} 43$ complexes. This segregation seems to be essential for mediating controlled cell death ${ }^{62}$.

Synthetic multivalent glycomaterials are being examined for antimicrobial therapy, anti-adhesive therapy, targeted drug delivery, cell separation and imaging purposes. One of the most impressive examples involves the design and chemical synthesis of an oligovalent starfish-shaped compound that has subnanomolar inhibitory activity for the B-5 subunit of Shiga-like toxin I (ref. 63). A co-crystal structure of the starfish-shaped saccharide ligand and Shiga-like toxin I showed that two trisaccharides at the tips of each of five spacer arms simultaneously engage all five B subunits of two toxin molecules.

Multivalent carbohydrate ligands have also been used for interfering in cell-signalling events ${ }^{59}$. For example, it has been shown that exposure of B-cells to polymers containing sialylated oligosaccharides and a ligand for B-cell receptor (BCR) attenuates key steps in BCR signalling events compared with a polymer containing BCR ligands alone $e^{64}$. The polymer containing BCR ligand alone clusters the BCRs, resulting in cellular activation and immune responses. However, the bifunctional polymer engages both the BCR and the immuno-suppressing co-receptor Siglec-2 (CD22), thereby dampening B-cell activation. Siglec- 2 is a member of the sialic acid-binding Ig-like lectin (Siglec) family of receptors, which can interact with glycoconjugates possessing terminal sialic acid residues. The results of this study indicate that trans-interactions between Siglec-2 on a B-cell and sialyated oligosaccharides on 


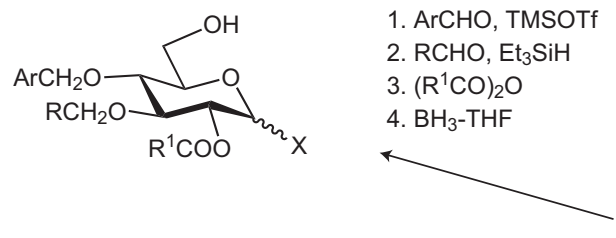

Overall yields

$50-95 \%$
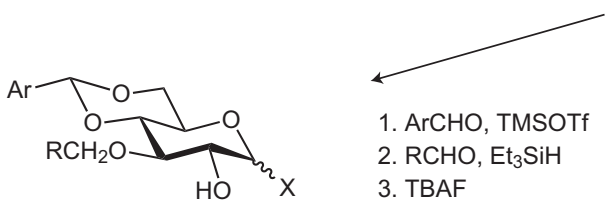

1. ArCHO, TMSOTf

2. $\mathrm{RCHO}, \mathrm{Et}_{3} \mathrm{SiH}$

3. TBAF

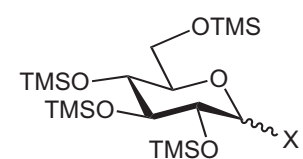

$X=\alpha$-OMe or $\beta$-STol

\section{ArCHO, TMSOTf \\ 2. $\mathrm{RCHO}, \mathrm{Et}_{3} \mathrm{SiH}$ \\ 3. $\left(\mathrm{R}^{1} \mathrm{CO}\right)_{2} \mathrm{O}$ \\ 4. $\mathrm{HCl}, \mathrm{NaCNBH}_{3}$}
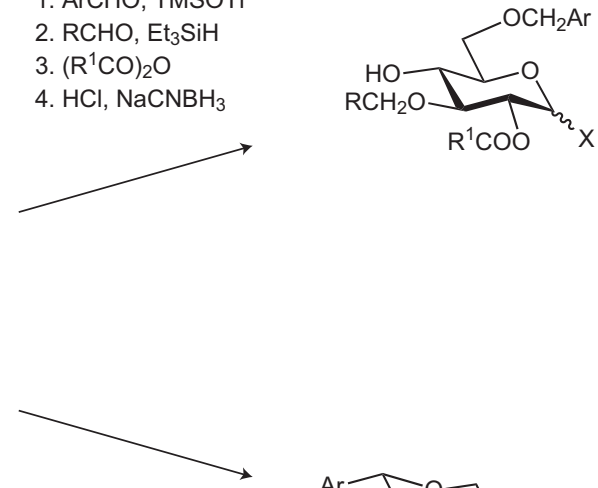

1. ArCHO, TMSOTf

2. $p$-OMePhCHO, $\mathrm{Et}_{3} \mathrm{SiH}$

3. TBAF

4. $\left(\mathrm{R}^{1} \mathrm{CO}\right)_{2} \mathrm{O}$

5. DDQ b

\begin{tabular}{|c|}
\hline $\begin{array}{l}\text { (2-1)- } \alpha \text {-D-mannoside } \\
\text { (3-1)- } \beta \text {-D-galactoside } \\
\text { (3-1)(6-1)- } \beta \text {-D-mannoside } \\
\text { (3-2)- } \beta \text {-D-galactoside } \\
\alpha \text {-D-mannoside } \\
\beta \text {-D-GlcNAc } \\
(6-2)-\beta \text {-D-galactoside } \\
(3-1)(4-1)-\beta \text {-D-GlcNAc } \\
(2-1)(4-1)-\beta \text {-D-mannoside } \\
(4-1)-\beta \text {-D-glucoside } \\
(2-1)-\beta \text {-D-galactoside } \\
(2-1)(6-1)-\beta \text {-D-mannoside }\end{array}$ \\
\hline
\end{tabular}

Abundance (\%)
$\begin{gathered}21.7 \\ 8.8 \\ 8.0 \\ 7.8 \\ 7.1 \\ 5.2 \\ 4.7 \\ 4.3 \\ 2.8 \\ 2.7 \\ 2.4 \\ 2.3 \\ 2.1 \\ 1.6 \\ 1.5 \\ 1.5\end{gathered}$

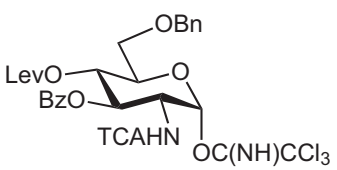

(4-1)- $\beta$-D-GIcNAc

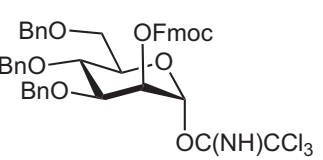

(2-1)- $\alpha-D-m a n n o s i d e$

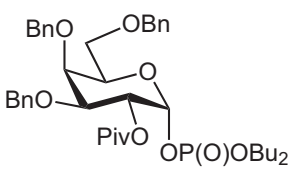

$\beta$-D-galactoside

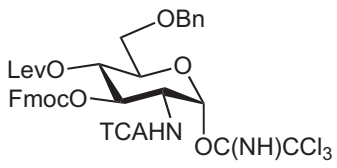

(3-1)(4-1)- $\beta$-D-GIcNAc

Figure 2 | Orthogonally protected mono- and disaccharide building blocks for oligosaccharide synthesis. a, One-pot protection of glucosides; with only four different types of one-pot reactions, a total of 152 different glucose building blocks can be reached. $\mathbf{b}$, The 15 most abundant monosaccharide units and their linkage types found in mammalian oligosaccharides are shown in the table. To the right is shown examples of four orthogonally protected building blocks that can be used to synthesize some of the units in the table. c, Disaccharide building blocks that can be used for a modular synthesis of heparan sulfate. Ac, acetyl; All, allyl; Bn, benzyl; Bz, benzoyl; DDQ, 2,3-dichloro-5,6-dicyanobenzoquinone; Et ${ }_{3} \mathrm{SiH}$, triethylsilane; GlcNAc, N-acetyl glucosamine; Lev, levolinoyl; Me, methyl; Piv, pivaloyl; TBAF, tetrabutylammonuium fluoride; TCA, trichloroacetyl; TMS, trimethylsilyl; TMSOTf, trimethylsilyl trifluoromethanesulfonate; Tol, toluyl.

another cell serves as an innate form of self-regulation thereby preventing auto-immune reactions.

\section{Streamlining complex oligosaccharide synthesis}

The realization that complex oligosaccharides and glycoconjugates are involved in numerous biological processes and have the potential for drug and vaccine development has stimulated the development of chemical and enzymatic methods for the preparation of this class of compounds. However, unlike oligonucleotide and peptide synthesis, there are no general protocols for the preparation of complex carbohydrates of biological importance. As a result, the chemical synthesis of each target is a research project, which may take many months and sometimes years to be completed. However, in recent years, facile procedures for the preparation of properly protected monosaccharides, stable yet highly reactive leaving groups for the anomeric centre, and stereoselective glycosylation protocols, have been developed ${ }^{22-25,28,65}$. Furthermore, convergent synthetic strategies have become available that allow the convenient assembly of complex oligosaccharides from properly protected building units involving a minimum number of synthetic steps. Also, the repertoire for chemoselective strategies has been considerably expanded. The next sections highlight several chemical and enzymatic approaches that have the potential to streamline the process of oligosaccharide synthesis.

\section{Monosaccharide synthesis}

One-pot multistep procedures. The preparation of a monosaccharide building block for complex oligosaccharide assembly requires extensive protecting-group manipulations. Therefore, it is to be expected that streamlining these manipulations will greatly reduce the time and effort required for the synthesis of a complex oligosaccharide target. An example of such a process is a parallel combinatorial, sequential one-pot multistep procedure for the selective protection of monosaccharides ${ }^{66}$. This expedient methodology incorporates up to seven reaction steps, obviating the need to carry out intermittent tedious work-up and time-consuming purifications. Thus, several 2,3,4,6-tetra-O-trimethylsilylated glucopyranosides bearing an anomeric group could be transformed into a set of differentially protected C-2, C-3, C-4 and C-6 alcohols and fully 
protected monosaccharides in high yields (Fig. 2a). A key feature of the approach is that all protecting-group manipulations are catalysed by the Lewis acids, trimethylsilyl trifluoromethane sulfonate (TMSOTf) or $\mathrm{Cu}(\mathrm{OTf})_{2}$. The resulting tailor-made glycosyl donors and acceptors can then be used for oligosaccharide synthesis.

Monosaccharide building blocks for oligosaccharide assembly. To speed up the process of oligosaccharide synthesis, efforts are also underway to identify limited numbers of monosaccharide building blocks that can repeatedly be used for the synthesis of a wide range of target structures. For example, the Seeberger and von der Lieth laboratories have undertaken a bioinformatics analysis of a database of 3,299 oligosaccharide structures from 38 mammalian species ${ }^{67}$. It was found that the preparation of all oligosaccharides by a linear chemical synthetic approach would require as many as 224 different monosaccharide building blocks. However, the analysis also indicated that $88 \%$ of the glycosidic linkages can be constructed by only 20 different monosaccharide building blocks (Fig. 2b). Thus, twenty putative building blocks were designed that represent these most abundant linkages and for this purpose benzyl ethers (Bn) and pivaloyl (Piv), acetyl (Ac) and benzoyl (Bz) esters and trichlorocarbamates (TCA) were selected as permanent protecting groups and the 9-fluorenylmethyl carbonate (Fmoc) as a temporary protecting group. To install branched points, the levulinoyl ester (Lev) and p-methoxybenzyl (PMB) ether were proposed as additional temporary protecting groups.

Based on the analysis of the structural motifs of heparan sulfate, it has been proposed that only six strategically selected monosaccharides are required to prepare eight disaccharide motifs, which in principle should give access to all possible heparan sulfate structures $^{68}$. In this strategy, the Lev esters are employed for those hydroxyls that need sulfation. In heparan sulfate, the C-3 and C-6 of the glucosamine and C-2 hydroxyls of the glycuronic acid moiety can be sulfated and, therefore, depending on the sulfation pattern of a targeted disaccharide module, one or more of these positions will be protected as Lev esters (Fig. 2c). An important feature of the Lev ester is that when present at the C-2 position, it will be able to direct the formation of 1,2-trans-glycosides through neighbouring group participation (NGP). In case the C-2 position of a disaccharide module does not need sulfation, an acetyl group is employed as a permanent protecting group. This ester can also perform NGP and is stable under the conditions used for the removal of Lev esters. An azido group is used as an amino-masking functionality, which does not perform NGP and therefore allows the introduction of a-aminoglucosides. An azido-group can easily be reduced to an amine, which can either be acetylated or sulfated. The C-4 hydroxyl, which is required for extension, is masked using an Fmoc group. The Fmoc group can be selectively removed with $\mathrm{Et}_{3} \mathrm{~N}$ in dichloromethane without affecting the Lev ester, whereas the Lev ester can be cleaved with hydrazinium acetate without affecting the Fmoc carbonate $^{69}$. The anomeric centre of the disaccharides are protected as allyl glycosides, and this functionality can easily be removed by isomerization without affecting the other protecting groups. The resulting lactol can then be converted into a trichloroacetimidate by employing $\mathrm{K}_{2} \mathrm{CO}_{3}$ and trichloroacetonitrile in dichloromethane.

It is important to note that the success of these unified building block strategies relies heavily on the premise that each glycosylation will be successful. In practise, this will not be the case and it is to be expected that additional saccharide building blocks will be required to address possible synthetic difficulties. To address such potential difficulties, sets of orthogonal protecting groups are being developed $^{70}$ that provide additional synthetic flexibility as they offer the possibility to change the order of glycosylation.

Anomeric control in glycosylations

Neighbouring group participation. The stereoselective introduction of glycosidic linkages (Fig. 3a) is one of the most challenging aspects in complex oligosaccharide synthesis ${ }^{25}$. The nature of the protecting group at C-2 of a glycosyl donor is a major determinant of the anomeric selectivity. A protecting group at C-2, which can perform NGP during a glycosylation, will give 1,2-trans glycosides (Fig. 3b). In these reactions, a promoter activates an anomeric leaving group resulting in its departure and the formation of an oxacarbenium ion. Subsequent NGP by the $2-\mathrm{O}$-acyl protecting group will give a more stable five-membered dioxolenium-ion, which can only be formed as a 1,2-cis fused ring system. An alcohol can attack the anomeric centre of the dioxolenium-ion from only one face providing a 1,2-trans-glycoside. Thus, in the case of glucosyl-type donors, $\beta$-linked products will be obtained whereas mannosides will give $\alpha$-linked products.

1,2-Cis-glycosides can be obtained when a non-assisting functionality is present at C-2. In this type of glycosylation reaction, the anomeric selectivity will be determined by conditions such as solvent, temperature and promoter, as well as the constitution of the glycosyl donor and acceptor (for example, type of saccharide, leaving group at the anomeric centre, protection and substitution pattern). Despite extensive optimization efforts, the introduction of 1,2-cis glycosides often leads to mixtures of anomers.

The recent introduction of a new glycosylation approach based on NGP by an (S)-(phenylthiomethyl)benzyl moiety at C-2 of a glycosyl donor (Fig. 3c) offers an exciting strategy for the introduction of 1,2-cis-glucosides and 1,2-cis-galactosides ${ }^{71}$. On formation of an oxa-carbenium ion, the nucleophilic phenylthio moiety of the C-2 functionality will participate, leading to the formation of an intermediate sulfonium ion as either a trans- or cis-decalin. The formation of the trans-decalin is strongly favoured because of the absence of unfavourable gauche interactions. In addition, the cisdecalin system will place the phenyl-substituent in an axial position inducing further unfavourable steric interactions. Displacement of the equatorial anomeric sulfonium ion by a sugar alcohol will then lead to the formation of a 1,2-cis glycoside. Indeed, NMR experiments have convincingly shown the selective formation of a $\beta$-linked sulfonium ion intermediate. The $(S)$-(phenylthiomethyl) benzyl moiety could readily be introduced by reaction of a sugar alcohol with $(S)$-(phenylthiomethyl)benzyl acetate in the presence of $\mathrm{BF}_{3}-\mathrm{OEt}_{2}$ and removed by conversion into acetyl ester by treatment with $\mathrm{BF}_{3}-\mathrm{OEt}_{2}$ in acetic anhydride. Both reactions proceed through an intermediate episulfonium ion.

Intramolecular aglycon delivery. Another innovative approach, which has been successfully employed for the preparation for $\beta$-mannosides, involves intramolecular aglycon delivery (IAD; Fig. 3d $)^{72,73}$. $\beta$-Mannosides, which are 1,2-cis glycosides, are difficult to introduce owing to the axial C-2 substituent, which sterically blocks incoming nucleophiles from the $\beta$-face, and the $\Delta$-anomeric effect, which provides additional stabilization of the $\alpha$-anomer ${ }^{74,75}$. In the IAD strategy, the sugar alcohol $(\mathrm{ROH})$ is linked via an acetal or silicon tether ( $\mathrm{Y}$ in Fig. $3 \mathrm{~d}$ ) to the C-2 position of a mannosyl donor. Subsequent activation of the anomeric centre of this adduct forces the aglycon to be delivered from the $\beta$-face of the glycosyl donor. IAD has also been employed for the instalment of $\alpha$-D-glucosides, $\beta$-L-rhamnopyranosides, $\beta$-D-arabinofuranosides, $\beta$-D-fructofuranosides, $\alpha$-D-fucofuranoside and $\alpha, \alpha$ trehaloses such as sulfolipid-1 (SL-1). The synthesis of SL-1 by the Bertozzi group involved oxidative coupling of a glucosyl acceptor carrying an a-anomeric 3,4-dimethoxybenzyl ether, with a thioglycosyl donor to produce the mixed acetal (inverse tethering) ${ }^{76}$. Ensuing glycosylation using methyl trifluoromethanesulfonate (MeOTf) as promoter gave only the $a, a$ trehalose product in good yield.

Anomeric control by electronic and steric effects. Crich and coworkers have pioneered an attractive approach for the introduction of $\beta$-mannosides by the in situ formation of an $\alpha$-anomeric 


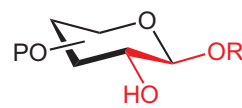

1,2-Trans gluco-type

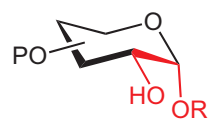

1,2-Cis gluco-type

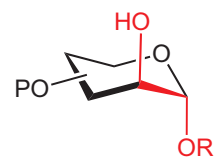

1,2-Trans

manno-type

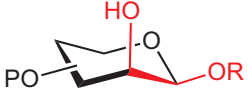

1,2-Cis

manno-type

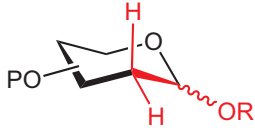

2-Deoxy

glycoside

b<smiles>[R]C(=O)O[C@H]1OCC[C@@H](O)C[C@@H]1I</smiles>

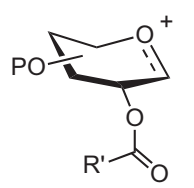

Oxa-carbenium ion

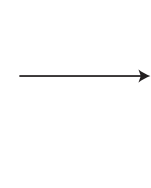

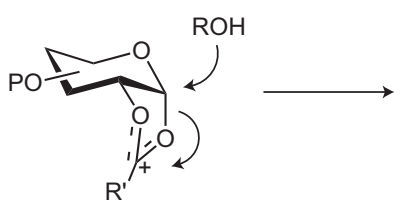

Dioxolenium ion

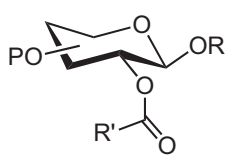

1,2-Trans-glycoside

c

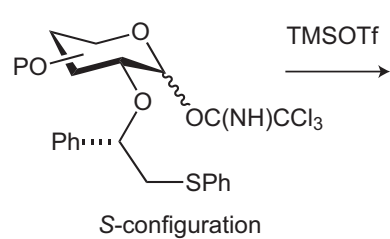

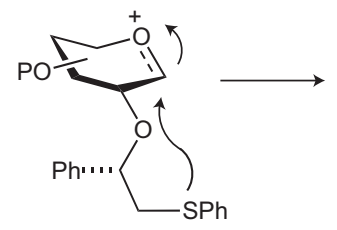

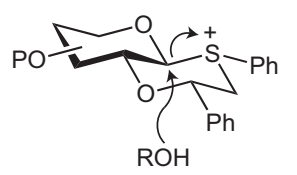

$\beta$-Sulfonium ion

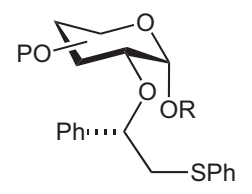

1,2-Cis-glycoside

d

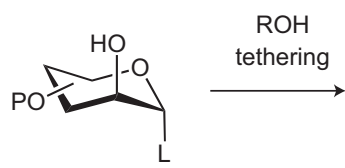

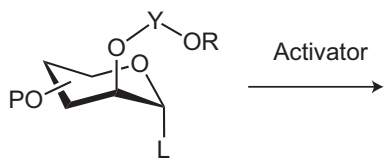<smiles>[R6]O[C@@H]1CC[C@H]2O[C@H]1[O+]([R])O2</smiles>

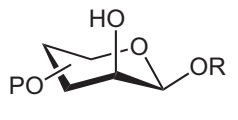

e
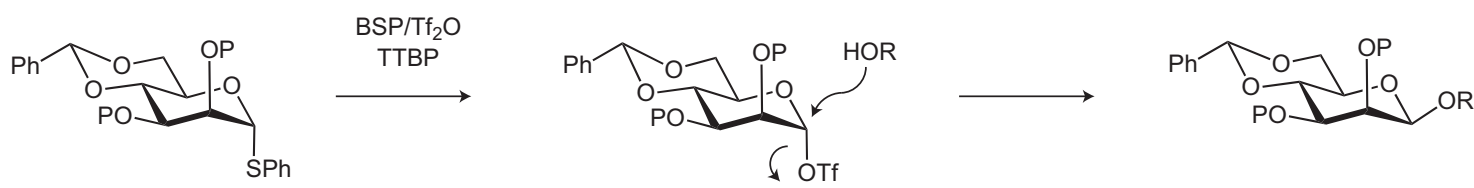

Figure 3 | Common methods for the stereoselective formation of glycosidic linkages. a, Different types of anomeric linkages. $\mathbf{b}$, Preparation of 1,2-trans glycosides by C-2 NGP. c, Chiral auxiliary controlled installation of 1,2-cis-glycosides. A stable $\beta$-sulfonium ion shields the $\beta$-face, forcing the incoming alcohol to attack from the $\alpha$-face. $\mathbf{d}$, Synthesis of $\beta$-mannosides by intramolecular aglycon delivery. The alcohol is tethered to the $\mathrm{C}-2$ position; subsequent activation of this adduct forces the alcohol to be delivered from the $\beta$-face. $Y=\mathrm{CH}_{2}, \mathrm{SiMe}_{2}$, 4-methoxybenzylidene, 2-naphthylmethylidene or 3,4-dimethoxybenzylidene). e, Introduction of $\beta$-mannosidic linkages by in situ formation of an $\alpha$-triflate that is displaced in a $S_{N} 2$-like manner to form a $\beta$-mannoside. $R$, glycosyl, alkyl or aryl; $\mathrm{P}$, protecting group; $\mathrm{BSP}$, 1-benzenesulfinyl piperidine; $\mathrm{TTBP}$, tri-tert-butylpyrimidine; $\mathrm{Tf}_{2} \mathrm{O}$, trifluoromethanesulfonic anhydride.

triflate (Fig. 3e) $)^{75,77,78}$. For example, treatment of a thioglycoside with 1-benzenesulfinyl piperidine (BSP) and trifluoromethanesulfonic anhydride $\left(\mathrm{Tf}_{2} \mathrm{O}\right)$ results in the formation of an $\alpha$-triflate because of a strong endo-anomeric effect. An $\mathrm{S}_{\mathrm{N}} 2$ like-displacement of the a-triflate by a sugar hydroxyl will then result in the formation of a $\beta$-mannoside. A prerequisite of $\beta$-mannoside formation is that the donor is protected by a 4,6-O-benzylidene acetal. It has been proposed that this protecting group opposes oxa-carbenium formation $\left(\mathrm{S}_{\mathrm{N}} 1\right.$ glycosylation) due to the torsional strain engendered by the half chair or boat conformation of this intermediate. Recently, Bols and co-workers proposed an alternative model by dissected electronic and torsional effects imposed by $4,6-O$-acetals ${ }^{79,80}$. Thus, O-6 can adopt three staggered conformations ( $t g, g t$ and $g g$ ), however, a 4,6-O-acetal forces the O-6 substituent in a $\operatorname{tg}$ conformation, which places its dipole anti-parallel to the electron-deficient anomeric centre formed in the transition state, thereby causing a destabilizing effect. In the $g g$ and $g t$ conformation the dipoles are perpendicular to the developing positive charge and thus less destabilizing. The electronic disarming effect was found to be approximately equal to the torsional effect of a 4,6-O-benzylidene acetal.

Innovative approaches have been developed for controlling 1,2-cis anomeric selectivities by imposing steric or conformational constraints. For example, glycosylations with galactosyl donors protected with a 4,6-O-di-tert-butylsilyl (DTBS) group gave the corresponding $\alpha$-galactosides with very high $\alpha$-anomeric selectivities, even in the presence of a C-2 group that can perform NGP (Fig. 4a). Probably, the bulky DTBS group blocks the $\beta$-face of the galactoside, forcing the incoming nucleophile to approach from the $\alpha$-face.

Factors such as small differences between anomeric effects of the $\alpha$ - and $\beta$-anomers and inherent ring flexibilities have complicated the development of a general method for the stereoselective introduction of 1,2-cis-furanosides. It has been reasoned that by examining possible conformers of the arabinofuranosyl oxa-carbenium ion, it might be possible to identify one that favours attack from the $\beta$-face (a 1,2-cis attack) (Fig. 4b) ${ }^{81}$. In this respect, oxa-carbenium ions of furanosides can adopt two possible low-energy conformations in which $\mathrm{C}-3$ is either above $\left({ }^{3} \mathrm{E}\right)$ or below the plane $\left(\mathrm{E}_{3}\right)$ of C-4, O-(endo), C-1 and C-2. The Newman projection of the $\mathrm{E}_{3}$ conformer of $\mathrm{D}$-arabinofuranose showed that nucleophilic attack from the $\alpha$-face is disfavoured because an eclipsed $\mathrm{H}-2$ will be encountered. On the other hand, an approach from the $\beta$-face was expected to be more favourable because it will experience only staggered substituents. Computational studies have indicated that the arabinofuranosyl oxa-carbenium ion can be locked in 
the $\mathrm{E}_{3}$ conformation by employing a 3,5-O-(di-tert-butylsilane)protecting group, which places $\mathrm{O}-5$ and $\mathrm{O}-3$ in a pseudo-equatorial orientation resulting in a perfect chair conformation of the protecting group. Indeed, an arabinofuranosyl donor protected by a DTBS group gave excellent $\beta$-selectivities in a range of glycosylations with glycosyl acceptors having primary and secondary alcohols. The method has been successfully employed for the synthesis of an arabinogalactan fragment derived from plant cell walls and for the preparation of arabinogalactan and lipoarabinomannan domains of Mycobacteria ${ }^{82}$.

Sialic acid glycosylations. Sialic acids are a family of naturally occurring 2-keto-3-deoxy-nononic acids that are involved in a wide range of biological processes and thus far 50 different derivatives have been reported. The C-5-amino derivative represents the long-known neuraminic acid and its amino function can either be acetylated (Neu5Ac) or glycolylated (Neu5Gc). The hydroxyls of these derivatives can be further acetylated, most commonly at C-9 but di- and tri-Oacetylated derivatives are also known. The use of glycosyl donors of sialic acid is complicated by the fact that no C-3 functionality is present to direct the stereochemical outcome of glycosylations ${ }^{27}$. The deoxy moiety in combination with the electron-withdrawing carboxylic acid at the anomeric centre makes these derivatives prone to elimination. Also, glycosylations of Neu5Ac take place at a sterically hindered tertiary oxa-carbenium ion intermediate.

Recently, the direct and stereoselective introduction of a-sialosides has been achieved by acylation of the acetamido group of a corresponding donor. This modification results in dramatic increases in reactivity and $\alpha$-anomeric selectivity (Fig. 4c). The first compound of this class was a 2-thioglycosyl donor of Neu5Ac bearing a di- $N$-acetyl ( $N$-acetylacetamido) functionality at C-5 (ref. 83). Further improvements came with the use of azido, $\mathrm{NH}$-trifluoroacetyl (TFA), $\mathrm{NH}$-2,2,2-trichloroethoxycarbonyl (Troc), $\mathrm{NH}$-Fmoc, $\mathrm{NH}$-trichloroacetyl, $\mathrm{N}$-phthalimide (Phth) and

a
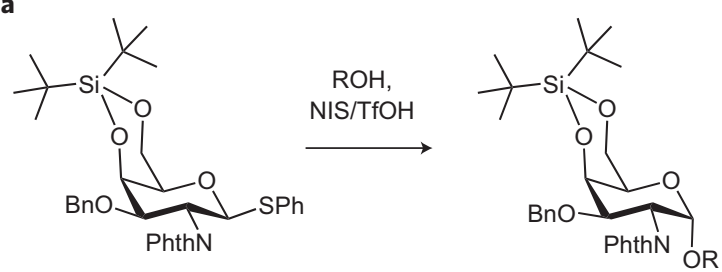

c

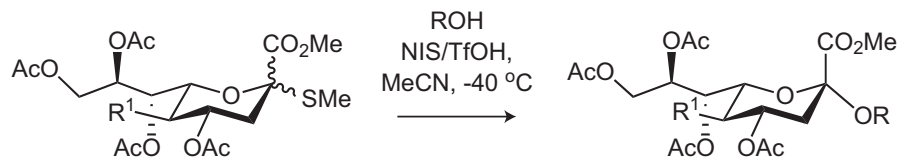

$\mathrm{R}^{1}=\mathrm{NAc}_{2}, \mathrm{NHTroc}, \mathrm{NHTFA}, \mathrm{NHFmoc}, \mathrm{NPhth}, \mathrm{N}_{3}$ or $\mathrm{CBz}$
$5 N, 4 O$-carbonyl groups, and it has been shown that this type of protection improved the efficiency of $\alpha$-selective sialylation providing easy access to various $\alpha(2,3), \alpha(2,6)-, \alpha(2,8)$ - and $\alpha(2,9)$-linked sialosides ${ }^{84}$. The introduction of $5 \mathrm{~N}, 4 \mathrm{O}$-carbonyl-protected sialyl donors have made it feasible to prepare sialosides by one-pot multistep glycosylation protocols..$^{85-87}$

Stereoselective introduction of 2-deoxy-2-amino-glycosides. 2-Deoxy-2-amino-glycosides are common constituents of $\mathrm{N}$ - and $O$-linked glycoproteins, glycolipids and proteoglycans. $\beta$-Linked 2-deoxy-2-amino-glucosides and galactosides can easily be obtained by employing an amino-protecting group that can perform NGP such as Phth, tetrachlorophthalimido (TCP), $N$-dimethyl maleoyl, trichloroacetyl, allyloxycarbonyl and Troc ${ }^{23}$.

2-Deoxy-2-azido-glucosides and galactosides are commonly employed for the introduction of 1,2-cis-glycosides. In this respect, the azido moiety is a non-participating functionality that is stable under a wide variety of reaction conditions but can readily be reduced to an amine with reagents such as phosphines and thiols, and by catalytic hydrogenation. Invariably, the use of 2-azido-2-deoxy-glycosyl donors leads to the formation of mixtures of anomers, which requires time-consuming purification protocols resulting in loss of material. However, it has been found that TMSOTf-promoted glycosylations of 2-azido-2-deoxy-glucosyl trichloroacetimidates provide excellent $a$-anomeric selectivities when performed at a relatively high reaction temperature in the presence of PhSEt or thiophene ${ }^{88}$. NMR spectroscopy and computational studies have indicated that these glycosylations proceed through an equatorial anomeric sulfonium ion, which on displacement by a sugar alcohol provides an axial glycoside.

It has also been found that 2,3-oxazolidone-protected glucosyl donors provide excellent $a$-anomeric selectivity when a sufficient amount of acid promoter is employed (Fig. 4d ${ }^{89-91}$. NMR-monitored glycosylation and anomerization experiments have shown that

b
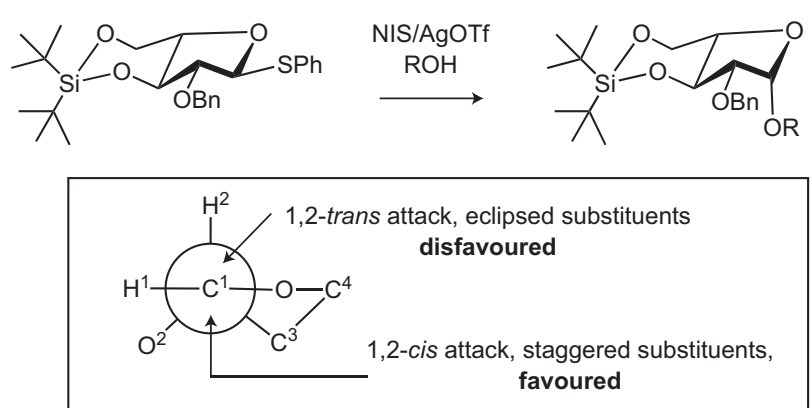

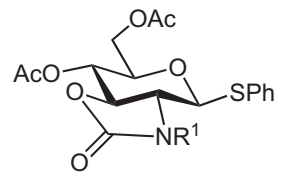
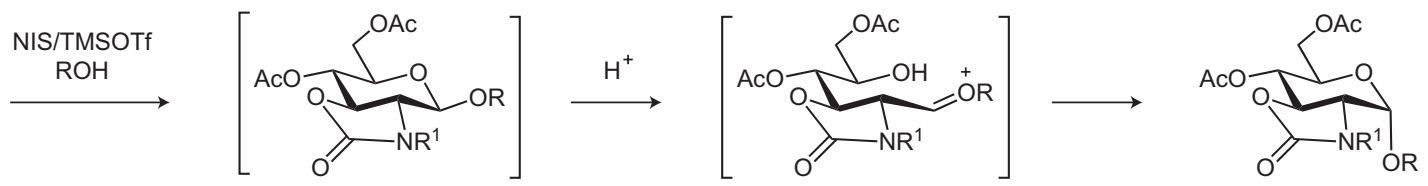

Figure 4 | Further methods for the stereoselective formation of glycosidic linkages. a, Formation of an $\alpha$-galactoside by steric constraints induced by a 4,6-O-silyl acetal. $\mathbf{b}$, Introduction of $\beta$-arabinosidic linkages by locking the five-membered ring in a conformation that favours an attack of the incoming nucleophile from the 1,2-cis face. The panel at the bottom shows the Newman projection of the locked conformer. c, Introduction of $\alpha$-sialosides by appropriate protection of the $\mathrm{N}$-acetamido moiety. d, Formation of a-linked 2-amino-2-deoxy-glucosides using 2,3-oxazolidone protected glycoside donors. CBz, benzyloxycarbonyl; NIS, N-iodosuccinimide; Phth, phthalimido; R, glycosyl, alkyl or aryl; TFA, trifluoroactyl; TfOH, trifluoromethanesulfonic acid; Troc, 2,2,2-trichloroethoxycarbonyl. 
initially a $\beta$-glycoside is formed that anomerizes by an intramolecular reaction involving an endocyclic $\mathrm{C}-\mathrm{O}$ bond cleavage, to give mainly or exclusively an a-glucoside. Probably, this mode of anomerization is promoted by ring strain induced by the cyclic protecting group. Evidence for endocyclic cleavage was obtained by intra- and intermolecular Friedel-Crafts reactions, chloride addition, and reduction of the generated cation ${ }^{92}$. Interestingly, it is important that pyranosides are locked in a chair conformation for endocyclic opening because pyranosides with the distorted conformations do not undergo this reaction.

\section{One-pot multistep glycosylations}

In the past decade, a variety of synthetic strategies have been developed to assemble complex oligosaccharides from carefully selected monosaccharide building blocks using a minimal number of manipulations. Among these strategies, one-pot multistep glycosylations, in which several glycosyl donors are sequentially reacted in the same flask, are very attractive and furnish target oligosaccharides without the need for protecting-group manipulation and intermediate isolation ${ }^{93-95}$. Although many variations of the one-pot strategy have been developed, there are three major concepts, namely, chemoselective, orthogonal and pre-activation glycosylation strategies ${ }^{26,28}$.

In chemoselective glycosylation strategies, glycosyl donors with decreasing anomeric reactivity are allowed to react sequentially. For example, detailed studies by Wong and co-workers have shown ${ }^{94}$ the following reactivity order of $\mathrm{C}-2$ protecting groups on a thiogalactosyl donor: $\mathrm{N}_{3}<\mathrm{OClAc}<\mathrm{NPhth}<\mathrm{OBz}<\mathrm{OBn}$. The degree of deactivation also depends on the position of a protecting group and, for example, the following order has been found for a thiogalactoside modified by a benzoyl ester: C-4 > C-3 > C-2 > C-6. The anomeric reactivity can also be tuned by conformational constraints imposed by cyclic protecting groups and glycosylation conditions such as solvent and reaction temperature. The multiple ways of controlling anomeric reactivity has made it possible to perform several consecutive glycosylations without the need for intermediate work-up and product purification. For example, a three-component one-pot synthesis gave the GM1 oligosaccharide ${ }^{96}$, which is an epitope of small-cell lung cancer and a similar four-component one-pot synthesis afforded an $\alpha-G a l$ pentasaccharide ${ }^{97}$.

Orthogonal glycosylations use glycosyl donors and acceptors that have different anomeric groups (for example, $-\mathrm{F}$ and $-\mathrm{SR}$ ),

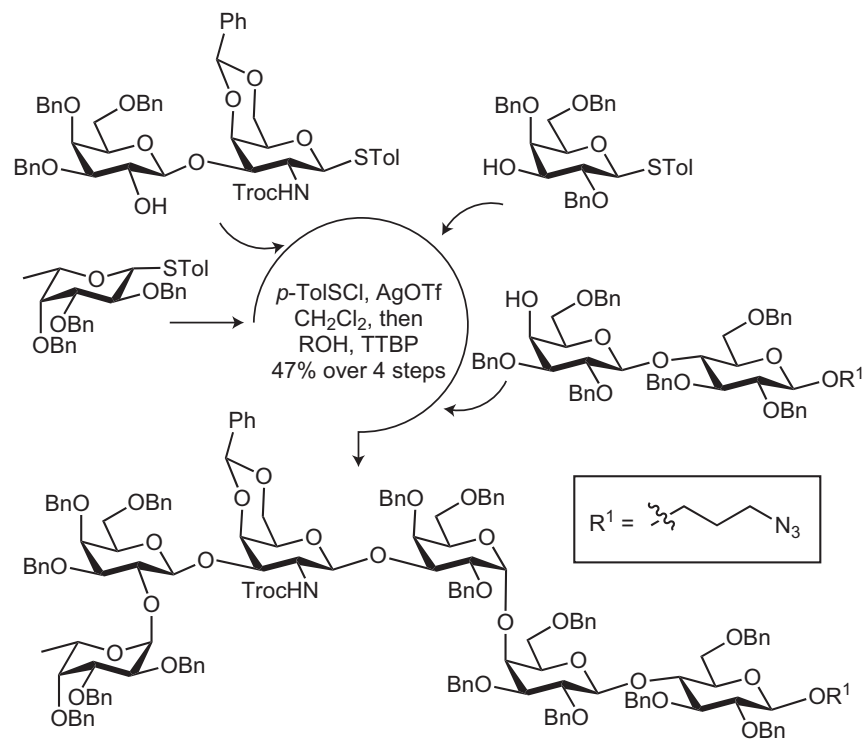

Figure 5 | One-pot chemical synthesis of the tumour-associated antigen Globo-H by iterative preactivation of p-tolyl thioglycosides. AgOTf, silver trifluoromethanesulfonate; $\mathrm{ROH}$, sugar alcohol. which can be activated without affecting the other one. These synthetic approaches are attractive because no or very few protecting-group manipulations are involved during the assembly of a complex oligosaccharide. Furthermore, it becomes feasible to condense saccharide building blocks independently of their relative anomeric reactivities ${ }^{98}$.

A flexible approach that integrates the advantages of both aforementioned strategies uses pre-activation of a glycosyl donor to generate a reactive intermediate in the absence of the acceptor. After the addition of the glycosyl acceptor, a disaccharide will be formed that has an identical leaving group at the reducing end. In the same reaction flask, the process of anomeric activation and glycosylation can be repeated to rapidly construct complex oligosaccharides. The successful implementation of the iterative one-pot glycosylation strategy requires, however, that a stoichiometric amount of promoter is completely consumed to prevent activation of a subsequent saccharide building block, the resulting intermediate should be sufficiently long-lived to allow the addition of the glycosyl acceptor, yet reactive enough for a high-yielding glycosylation, and side products should not interfere with the glycosylations. These requirements have been achieved by activation of thioglycosides with $p$-toluenesulfenyl triflate ( $p$ TSOTf) to give an anomeric triflate, which on addition of a thioglycosyl acceptor will give a glycoside product. The methodology has been applied to the preparation of several oligosaccharides of biological importance and, for example, the tumour-associated antigen Globo-H was assembled from three thioglycosyl building blocks by a one-pot procedure (Fig. 5) ${ }^{99}$.

\section{Polymer-supported oligosaccharide synthesis}

Encouraged by the successes of polymer-supported peptide synthesis, the first attempts at solid-phase oligosaccharide synthesis were reported in the 1970s, however, these efforts were futile because of a lack of efficient glycosylation methods. The past decade has seen a renewed interest in polymer-supported carbohydrate synthesis and different polymer support materials, linkers, synthetic strategies and glycosylating agents have been explored ${ }^{100,101}$.

Several oligosaccharides have been successfully prepared by polymer-supported approaches. For example, the Seeberger laboratory has reported the automated synthesis of a $\mathrm{Le}^{y}-\mathrm{Le}^{\mathrm{x}}$ nonasaccharide, which is a tumour marker that is being explored for the development of a cancer vaccine (Fig. 6) ${ }^{100}$. The nonasaccharide was synthesized by employing five monosaccharide building blocks and a polystyrene resin modified with an octenediol linker. Fmoc carbonates and Lev esters served as a versatile set of temporary protections facilitating the introduction of branching points. The cleavage of the Fmoc was achieved by three exposures to piperidine (20\% in DMF). Following each exposure, the solution from the reaction vessel was collected for UV analysis. Removal of the C-4 Lev ester from the glucosamine moiety was achieved by three exposures to a solution of hydrazine ( $10 \%$ in DMF). The solid-phase synthesis of the $\mathrm{Le}^{\mathrm{y}}-\mathrm{Le}^{\mathrm{x}}$ nonasaccharide was completed in 23 hours. The fully assembled oligosaccharide could be cleaved from the solid support by olefin cross-metathesis using a Grubbs catalyst under an ethylene atmosphere. The resulting anomeric pentenyl glycoside can be activated for glycosylation, hydrolysed or functionalized by radical-initiated addition of a thiol linker ${ }^{102}$.

Despite these successes, a general solution for routine and automated oligosaccharide synthesis remains to be established because of problems such as the need for large excesses of glycosyl donors, lack of anomeric control when 1,2-cis-glycosides need to be installed, unpredictability of glycosylations, and the additional steps required for linker funtionalization and protecting-group removal. However, as highlighted above, progress is being made in these areas, bringing the promise of automated oligosaccharide synthesis closer.

To address the need for a large excesses of glycosyl donor in polymer-supported oligosaccharide synthesis, solution-based 
a
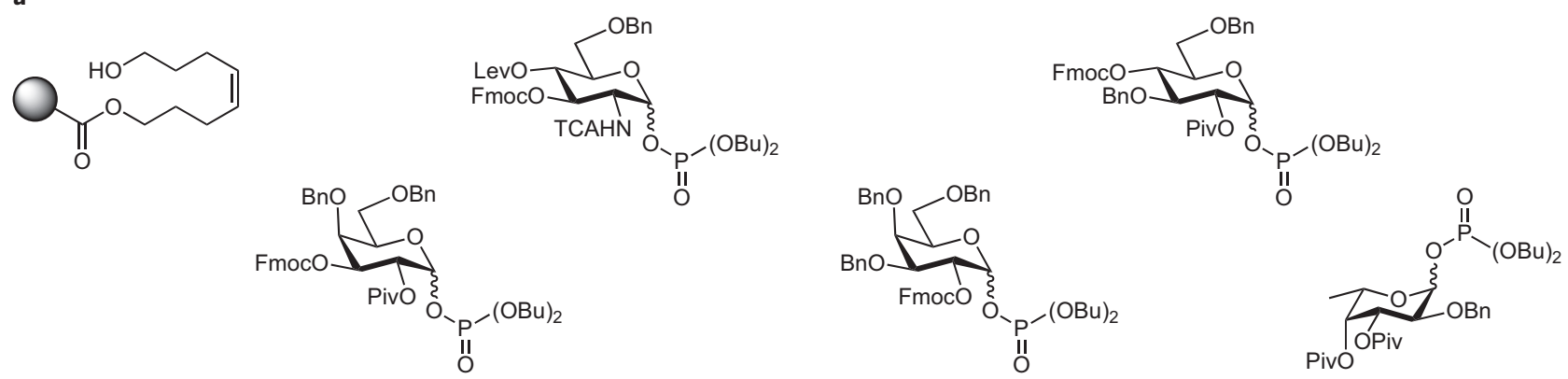

b

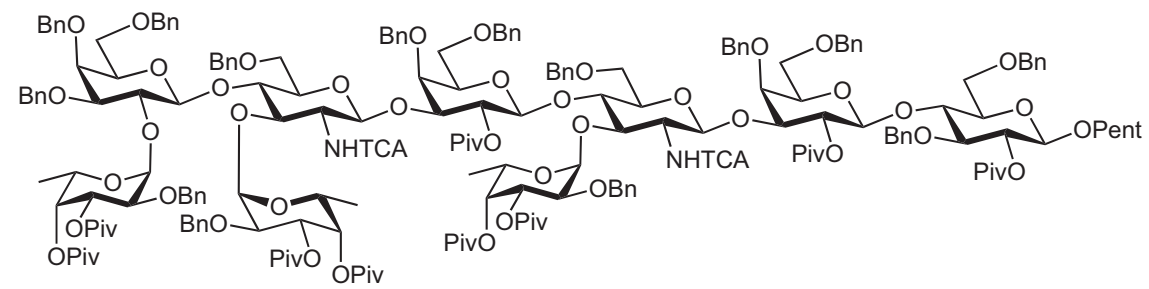

Figure 6 | Polymer-supported synthesis of the tumour-associated KH-1 antigen. a, The linker-modified polystyrene resin and orthogonally protected

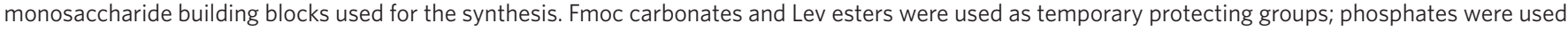
as anomeric leaving groups. b. The fully protected, resin-released, nonasaccharide antigen. Bu, butyl; Pent, pentenyl.

strategies have been developed in which the growing oligosaccharide chain is modified by a tag that allows selective precipitation, extraction or absorption for convenient purification. In particular, the use of light fluorous tags $\left(\mathrm{C}_{8} \mathrm{~F}_{17}\right)$ shows promise as it makes it possible to conduct protecting-group manipulations and glycosylations under conditions typically used for solution-phase chemistry ${ }^{103,104}$. However, tagged products can selectively be captured by filtration of the crude product through a fluorous solid-phase extraction column and then released by elution with methanol.

The technology has been employed for the synthesis of a mannooligosaccharide that has an $\alpha(1-6)$-backbone branched with $\alpha(1-3)$ mannosides ${ }^{103}$. The compound was assembled by using two mannosyl building blocks modified by a Lev ester and/or a tertiary butyldiphenylsilyl ether as temporary protecting groups for installing the branching point. Neighbouring-group participation by an acetyl or pivaloyl ester controlled the $\alpha$-anomeric selectivity. Advantageous features of the approach include the requirement of only a small excess of glycosyl donor to drive couplings to completion, ease of purification by solid extraction, the intermediates can be characterized by NMR spectroscopy (tags are invisible in ${ }^{1} \mathrm{H}$ NMR spectra) and the fluorous tags themselves are relatively unreactive. It has been shown that the fluorous solvophobic effect is sufficiently strong to adhere fluorous-tagged monosaccharides to a $\mathrm{C}_{8} \mathrm{~F}_{17}$-modified glass surface in defined locations, and is ideally suited for the generation of glycan arrays ${ }^{105}$.

\section{Enzymatic and chemoenzymatic approaches}

The need for efficient approaches to oligosaccharide synthesis has stimulated the development of chemo-enzymatic methods. Two basic methods have been developed that employ glycosyl transferases or the reverse activity of glycosyl hydrolases ${ }^{106}$.

Glycosyl transferases are essential enzymes for oligosaccharide biosyntheses and transfer a sugar residue from a sugar-nucleotide mono- and di-phosphate to a maturing oligosaccharide chain ${ }^{107}$. These enzymes are highly regio- and stereoselective and can be obtained by cloning and over-expression. The use of these enzymes combined with sophisticated methods to regenerate sugar nucleotides ${ }^{108,109}$ has led to the preparation of complex oligosaccharides and glycopeptides. For example, the methodology has been employed to construct a range of glycopeptides derived from the $N$-terminus of P-selectin glycoprotein ligand-1 (PSGL-1) ${ }^{110,111}$. This adhesion protein is expressed on white blood cells and has an important role in recruitment of these cells to inflamed tissue $e^{112}$. The synthetic glycopeptides provided valuable tools for pinpointing the structural characteristics necessary for interactions with the counter-part receptor, L-selectin. Recently, Nishimura and co-workers reported the combined use of microwave-assisted glycopeptide synthesis and glycosyltransferase-catalysed glycan extension in their impressive synthesis of a MUC-1 peptide carrying five sialylated hexasaccharides ${ }^{113}$. This combination strategy holds great promise for high-speed parallel syntheses of glycopeptide libraries.

Despite these successes, the scope of oligosaccharide synthesis by glycosyltransferases is still restricted owing to a lack of readily available glycosyltransferases and the high substrate specificities of many of these enzymes. It has, however, been recognized that glycoysl transferases from microbial sources may be rather promiscuous, making it possible to synthesize a range of oligosaccharide analogues. For example, Chen and co-workers have established a convenient one-pot, three-enzyme chemo-enzymatic approach for the synthesis of sialosides containing naturally occurring as well as non-natural sialic acid modifications at C5, C7, C8 and/or C9 (Fig. $7 \mathrm{a})^{114}$. In this method, $\mathrm{N}$-acetyl mannosamine or mannose is chemically modified and then converted into sialic acid derivatives using sialic acid aldolase from E. coli K-12. These compounds have been activated by a CMP-sialic acid synthetase from $N$. meningitides and then transferred to a range of suitable acceptors by an a $(2,6)$-sialyltransferase from Photobacterium damsela and an $\alpha(2,3)$-sialyltransferase from Paseurella multocida (Pm). The methodology was used to prepare 72 biotinylated $a(2,6)$-sialosides in 96-well plates from four biotinylated sialyltransferase acceptors and 18 sialic acids ${ }^{115}$. High-throughput screening assays performed in NeutrAvidin-coated microtiter plates show that, whereas the Sambucus nigra lectin binds to a2,6-linked sialosides with high promiscuity, human Siglec-2 is highly selective for a number of sialic acid structures and the underlying glycans. 
a

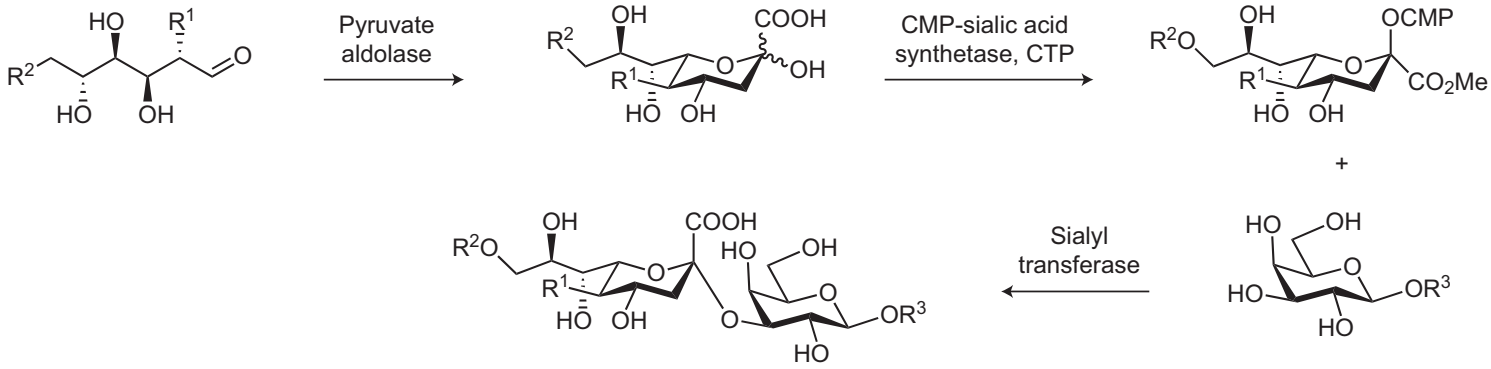

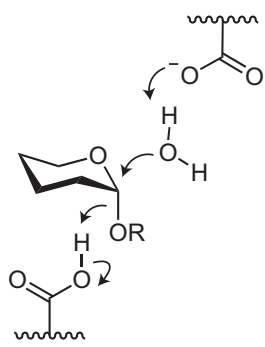

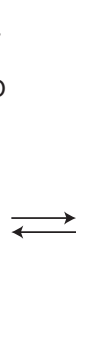

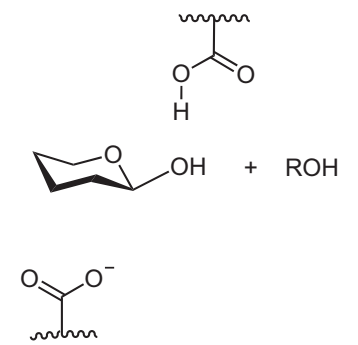
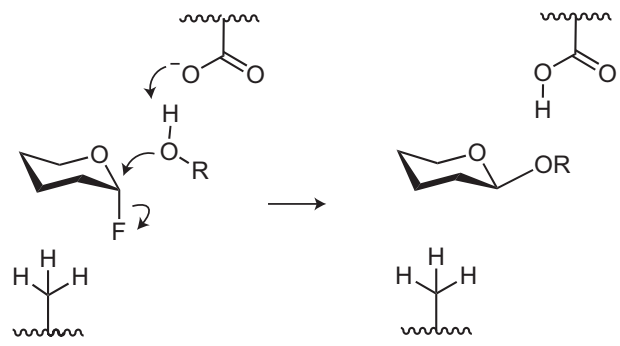

Figure 7 | Enzymatic and chemoenzymatic synthesis of oligosaccharides. a, Synthesis of a sialoside library using promiscuous enzymes, chemically modified $\left(R^{1}\right.$ and $R^{2}$ ) mannosamine and mannose, and modified galactose $\left(R^{3}\right)$ acceptors in a one-pot, three-enzyme approach. $\mathbf{b}$, Mechanism of inverting glycosidases for the degradation of oligosaccharides. $\mathbf{c}$, Mechanism for glycosylation by a glycosynthase using activated glycosyl fluorides as substrates. CMP, cytidine-5'-monophosphate; CTP, cytidine-5'-triphosphate.

Nature uses glycosyl hydrolases for the degradation of oligosaccharides (Fig. 7b). However, the reverse hydrolytic activity of these enzymes can be exploited in glycosidic bond formation. These approaches have, however, relatively low yields owing to the challenge of driving reactions in a thermodynamically unfavourable direction and because of enzymatic degradation of the reaction product. Withers and co-workers have addressed these problems by introducing 'glycosynthases', which are glycosidases rendered hydrolytically incompetent through the replacement of a nucleophilic aspartic or glutamic acid with another amino acid (Fig. $7 \mathrm{c})^{116}$. When supplied with glycosyl fluoride substrates of the opposite anomeric configuration to that of the natural substrate, the enzyme is often able to transfer this activated glycosyl donor to a suitable acceptor. For example, $\beta(1,4)$-mannans, which are major plant cell-wall polysaccharides representing a considerable synthetic challenge, have been prepared by employing a mutant endo- $\beta$-mannanase and an $\alpha$-mannobiosyl fluoride as glycosyl donor ${ }^{117}$. Also, a glycosynthase for the synthesis of $\beta$-linked glucuronic and galacturonic acid conjugates has been developed; such an enzyme has the potential to be used for the synthesis of a number of important oligosaccharides, such as plant and bacterial cell-wall components and mammalian glycosaminoglycans, such as heparan sulfate. Recent results indicate that the catalytic activities and substrate promiscuities of glycosynthases can be improved by directed evolution.

Well-defined glycoproteins have also been prepared by a chemoenzymatic approach whereby a glycan is transferred to a GlcNAc acceptor by the reverse hydrolytic activity of endo- $\beta-N$ acetylglucosaminidases (ENGases). This transfer reaction is particularly efficient when oxazolines are used as glycosyl donors ${ }^{118}$. The oxazolines are presumed mimics of the oxazolinium ion intermediate formed during the transfer reaction. The approach gave improved yields and, in addition, a broader range of substrates could be used as glycosyl donors, indicating that oligosaccharide oxazolines are more kinetically favoured substrates than the natural $\mathrm{N}$-glycans. In line with this work, Endo-M mutants, with diminished hydrolytic activity and intact transglycosylation activity for full-size $\mathrm{N}$-glycan oxazolines, are being developed ${ }^{119}$.

\section{Conclusions}

The development of routine procedures for the chemical synthesis of oligonucleotide fragments (DNA and RNA) and peptides has changed the face of modern biology. Unfortunately, no general methods are available for the preparation of complex carbohydrates of biological importance, which is hampering progress in glycobiology and glycomedicine. Recent developments such as onepot multistep protecting-group manipulations, the use of unified monosaccharide building blocks, the introduction of steroselective glycosylation protocols, one-pot multistep, polymer-supported and chemoenzymatic approaches for oligosaccharide assembly, are addressing these problems. Collectively, these methods have the promise to offer routine approaches for automated synthesis of wide ranges of oligosaccharides and glycoconjugates. The power of synthetic carbohydrate chemistry has been highlighted by the development of vaccines and anticoagulants for human use and the recent chemical synthesis of glycoproteins.

\section{References}

1. Varki, A. Biological roles of oligosaccharides - all of the theories are correct. Glycobiology 3, 97-130 (1993).

2. Bucior, I. \& Burger, M. M. Carbohydrate-carbohydrate interactions in cell recognition. Curr. Opin. Struct. Biol. 14, 631-637 (2004).

3. Ohtsubo, K. \& Marth, J. D. Glycosylation in cellular mechanisms of health and disease. Cell 126, 855-867 (2006).

4. Hart, G. W., Housley, M. P. \& Slawson, C. Cycling of O-linked beta- $\mathrm{N}$-acetylglucosamine on nucleocytoplasmic proteins. Nature 446, 1017-1022 (2007).

5. Bishop, J. R., Schuksz, M. \& Esko, J. D. Heparan sulphate proteoglycans finetune mammalian physiology. Nature 446, 1030-1037 (2007).

6. Kleene, R. \& Schachner, M. Glycans and neural cell interactions. Nature Rev. Neurosci. 5, 195-208 (2004).

7. Brockhausen, I. Mucin-type O-glycans in human colon and breast cancer: glycodynamics and functions. EMBO Rep. 7, 599-604 (2006).

8. Brown, J. R., Crawford, B. E. \& Esko, J. D. Glycan antagonists and inhibitors: a fount for drug discovery. Crit. Rev. Biochem. Mol. Biol. 42, 481-515 (2007).

9. Crocker, P. R., Paulson, J. C. \& Varki, A. Siglecs and their roles in the immune system. Nature Rev. Immunol. 7, 255-266 (2007).

10. Van Kooyk, Y. \& Rabinovich, G. A. Protein-glycan interactions in the control of innate and adaptive immune responses. Nature Immunol. 9, 593-601 (2008).

11. Freeze, H. H. Congenital disorders of glycosylation: CDG-I, CDG-II, and beyond. Curr. Mol. Med. 7, 389-396 (2007). 
12. Guo, H., Yi, W., Song, J. K. \& Wang, P. G. Current understanding on biosynthesis of microbial polysaccharides. Curr. Top. Med. Chem. 8, 141-151 (2008)

13. Trent, M. S., Stead, C. M., Tran, A. X. \& Hankins, J. V. Diversity of endotoxin and its impact on pathogenesis. J. Endotoxin Res. 12, 205-223 (2006)

14. Miller, S. I., Ernst, R. K. \& Bader, M. W. LPS, TLR4 and infectious disease diversity. Nature Rev. Microbiol. 3, 36-46 (2005).

15. Weidenmaier, C. \& Peschel, A. Teichoic acids and related cell-wall glycopolymers in Gram-positive physiology and host interactions. Nature Rev. Microbiol. 6, 276-287 (2008).

16. Sahly, H., Keisari, Y., Crouch, E., Sharon, N. \& Ofek, I. Recognition of bacterial surface polysaccharides by lectins of the innate immune system and its contribution to defense against infection: the case of pulmonary pathogens. Infect. Immun. 76, 1322-1332 (2008).

17. Vliegenthart, J. F. Carbohydrate based vaccines. FEBS Lett. 580, 2945-2950 (2006).

18. Pozsgay, V. Recent developments in synthetic oligosaccharide-based bacterial vaccines. Curr. Top. Med. Chem. 8, 126-140 (2008)

19. Nyame, A. K., Kawar, Z. S. \& Cummings, R. D. Antigenic glycans in parasitic infections: implications for vaccines and diagnostics. Arch. Biochem. Biophys. 426, 182-200 (2004).

20. Ada, G. \& Isaacs, D. Carbohydrate-protein conjugate vaccines. Clin. Microbiol. Infect. 9, 79-85 (2003).

21. Geyer, H. \& Geyer, R. Strategies for analysis of glycoprotein glycosylation. Biochim. Biophys. Acta 1764, 1853-1869 (2006).

22. Zhu, X. M. \& Schmidt, R. R. New principles for glycoside-bond formation. Angew. Chem. Int. Ed. 48, 1900-1934 (2009).

23. Bongat, A. F. G. \& Demchenko, A. V. Recent trends in the synthesis of O-glycosides of 2-amino-2-deoxysugars. Carbohydr. Res. 342, 374-406 (2007).

24. Buskas, T., Ingale, S. \& Boons, G. J. Glycopeptides as versatile tools for glycobiology. Glycobiology 16, 113R-136R (2006).

25. Demchenko, A. V. Stereoselective chemical 1,2-cis O-glycosylation: from 'sugar ray' to modern techniques of the 21st century. Synlett 1225-1240 (2003).

26. Codee, J. D. C., Litjens, R., van den Bos, L. J., Overkleeft, H. S. \& van der Marel, G. A. Thioglycosides in sequential glycosylation strategies. Chem. Soc. Rev. 34, 769-782 (2005).

27. Boons, G. J. \& Demchenko, A. V. Recent advances in O-sialylation. Chem. Rev. 100, 4539-4566 (2000).

28. Wang, Y. H., Ye, X. S. \& Zhang, L. H. Oligosaccharide assembly by one-pot multi-step strategy. Org. Biomol. Chem. 5, 2189-2200 (2007).

29. Blixt, O. et al. Printed covalent glycan array for ligand profiling of diverse glycan binding proteins. Proc. Natl Acad. Sci. USA 101, 17033-17038 (2004).

30. Paulson, J. C., Blixt, O. \& Collins, B. E. Sweet spots in functional glycomics. Nature Chem. Biol. 2, 238-248 (2006).

31. Stevens, J., Blixt, O., Paulson, J. C. \& Wilson, I. A. Glycan microarray technologies: tools to survey host specificity of influenza viruses. Nature Rev. Microbiol. 4, 857-864 (2006)

32. Petitou, M. \& van Boeckel, C. A. A synthetic antithrombin III binding pentasaccharide is now a drug! What comes next? Angew. Chem. Int. Ed. 43, 3118-3133 (2004)

33. De Kort, M., Buijsman, R. C. \& van Boeckel, C. A. A. Synthetic heparin derivatives as new anticoagulant drugs. Drug Discov. Today 10, 769-779 (2005).

34. Munoz, E. M. \& Linhardt, R. J. Heparin-binding domains in vascular biology. Arterioscler. Thromb. Vasc. Biol. 24, 1549-1557 (2004).

35. Liu, H., Zhang, Z. \& Linhardt, R. J. Lessons learned from the contamination of heparin. Nat. Prod. Rep. 26, 313-321 (2009).

36. Guerrini, M. et al. Oversulfated chondroitin sulfate is a contaminant in heparin associated with adverse clinical events. Nature Biotechnol. 26, 669-675 (2008)

37. Esko, J. D. \& Selleck, S. B. Order out of chaos: assembly of ligand binding sites in heparan sulfate. Annu. Rev. Biochem. 71, 435-471 (2002).

38. Capila, I. \& Linhardt, R. J. Heparin-protein interactions. Angew. Chem. Int. Ed. 41, 391-412 (2002).

39. Noti, C. \& Seeberger, P. H. Chemical approaches to define the structureactivity relationship of heparin-like glycosaminoglycans. Chem. Biol. 12, 731-756 (2005).

40. Rusnati, M., Oreste, P., Zoppetti, G. \& Presta, M. Biotechnological engineering of heparin/heparan sulphate: A novel area of multi-target drug discovery. Curr. Pharm. Des. 11, 2489-2499 (2005).

41. Rostand, K. S. \& Esko, J. D. Microbial adherence to and invasion through proteoglycans. Infect. Immun. 65, 1-8 (1997)

42. Avci, F. Y., Karst, N. A. \& Linhardt, R. J. Synthetic oligosaccharides as heparin-mimetics displaying anticoagulant properties. Curr. Pharm. Des. 9, 2323-2335 (2003).

43. Seeberger, P. H. \& Werz, D. B. Synthesis and medical applications of oligosaccharides. Nature 446, 1046-1051 (2007).

44. Wang, L. X. Toward oligosaccharide- and glycopeptide-based HIV vaccines. Curr. Opin. Drug. Discov. Devel. 9, 194-206 (2006)

45. Verez-Bencomo, V. et al. A synthetic conjugate polysaccharide vaccine against Haemophilus influenzae type b. Science 305, 522-525 (2004)

46. Slovin, S. F., Keding, S. J. \& Ragupathi, G. Carbohydrate vaccines as immunotherapy for cancer. Immunol. Cell Biol. 83, 418-428 (2005)

47. Galonic, D. P. \& Gin, D. Y. Chemical glycosylation in the synthesis of glycoconjugate antitumour vaccines. Nature 446, 1000-1007 (2007).
48. Cipolla, L., Peri, F. \& Airoldi, C. Glycoconjugates in cancer therapy. Anticancer Agents Med. Chem. 8, 92-121 (2008).

49. Buskas, T., Thompson, P. \& Boons, G. J. Immunotheraphy for cancer: synthetic carbohydrate-based vaccines. Chem. Commun. 5335-5349 (2009).

50. Barton, G. M. \& Medzhitov, R. Control of adaptive immune responses by Toll-like receptors. Curr. Opin. Immunol. 14, 380-383 (2002).

51. Rich, J. R. \& Withers, S. G. Emerging methods for the production of homogeneous human glycoproteins. Nature Chem. Biol. 5, 206-215 (2009).

52. Gamblin, D. P., Scanlan, E. M. \& Davis, B. G. Glycoprotein synthesis: an update. Chem. Rev. 109, 131-163 (2009).

53. van Kasteren, S. I. et al. Expanding the diversity of chemical protein modification allows post-translational mimicry. Nature 446, 1105-1109 (2007).

54. Yuan, Y. et al. Toward homogeneous erythropoietin: fine tuning of the C-terminal acyl donor in the chemical synthesis of the Cys(29)-Gly(77) glycopeptide domain. J. Am. Chem. Soc. 131, 5432-5437 (2009).

55. de la Fuente, J. M. \& Penades, S. Glyconanoparticles: types, synthesis and applications in glycoscience, biomedicine and material science. Biochim. Biophys. Acta 1760, 636-651 (2006).

56. Coullerez, G., Seeberger, P. H. \& Textor, M. Merging organic and polymer chemistries to create glycomaterials for glycomics applications. Macromol. Biosci. 6, 634-647 (2006).

57. Chabre, Y. M. \& Roy, R. Recent trends in glycodendrimer syntheses and applications. Curr. Top. Med. Chem. 8, 1237-1285 (2008).

58. Pieters, R. J. Maximising multivalency effects in protein-carbohydrate interactions. Org. Biomol. Chem. 7, 2013-2025 (2009).

59. Kiessling, L. L., Gestwicki, J. E. \& Strong, L. E. Synthetic multivalent ligands as probes of signal transduction. Angew. Chem. Int. Ed. 45, 2348-2368 (2006)

60. Mammen, M., Choi, S. K. \& Whitesides, G. M. Polyvalent interactions in biological systems: implications for design and use of multivalent ligands and inhibitors. Angew. Chem. Int. Ed. 37, 2755-2794 (1998)

61. Brewer, C. F., Miceli, M. C. \& Baum, L. G. Clusters, bundles, arrays and lattices: novel mechanisms for lectin-saccharide-mediated cellular interactions. Curr. Opin. Struct. Biol. 12, 616-623 (2002).

62. Pace, K. E., Lee, C., Stewart, P. L. \& Baum, L. G. Restricted receptor segregation into membrane microdomains occurs on human $\mathrm{T}$ cells during apoptosis induced by galectin-1. J. Immunol. 163, 3801-3811 (1999)

63. Kitov, P. I. et al. Shiga-like toxins are neutralized by tailored multivalent carbohydrate ligands. Nature 403, 669-672 (2000).

64. Courtney, A. H., Puffer, E. B., Pontrello, J. K., Yang, Z. Q. \& Kiessling, L. L. Sialylated multivalent antigens engage CD22 in trans and inhibit B cell activation. Proc. Natl Acad. Sci. USA 106, 2500-2505 (2009).

65. Codee, J. D. C. et al. A modular strategy toward the synthesis of heparinlike oligosaccharides using monomeric building blocks in a sequential glycosylation strategy. J. Am. Chem. Soc. 127, 3767-3773 (2005).

66. Wang, C. C. et al. Regioselective one-pot protection of carbohydrates. Nature 446, 896-899 (2007)

67. Werz, D. B. et al. Exploring the structural diversity of mammalian carbohydrates ("Glycospace") by statistical databank analysis. ACS Chem. Biol 2, 685-691 (2007)

68. Prabhu, A., Venot, A. \& Boons, G. J. New set of orthogonal protecting groups for the modular synthesis of heparan sulfate fragments. Org. Lett. 5, 4975-4978 (2003)

69. Zhu, T. \& Boons, G. J. A new set of orthogonal-protecting groups for oligosaccharide synthesis on a polymeric support. Tetrahedron: Asymmetry 11, 199-205 (2000).

70. Wong, C. H., Ye, X. S. \& Zhang, Z. Assembly of oligosaccharide libraries with a designed building block and an efficient orthogonal protection-deprotection strategy. J. Am. Chem. Soc. 120, 7137-7138 (1998).

71. Kim, J. H., Yang, H., Park, J. \& Boons, G. J. A general strategy for stereoselective glycosylations. J. Am. Chem. Soc. 127, 12090-12097 (2005).

72. Jung, K. H., Muller, M. \& Schmidt, R. R. Intramolecular O-glycoside bond formation. Chem. Rev. 100, 4423-4442 (2000).

73. Cumpstey, I. Intramolecular aglycon delivery. Carbohydr. Res. 343, 1553-1573 (2008)

74. Gridley, J. J. \& Osborn, H. M. I. Recent advances in the construction of beta-D-mannose and beta-D-mannosamine linkages. J. Chem. Soc., Perkin Trans. 1 2000, 1471-1491 (2000).

75. Cai, F., Wu, B. \& Crich, D. Stereocontrolled synthesis of mannans and rhamnans. Adv. Carbohydr. Chem. Biochem. 62, 251-309 (2009).

76. Leigh, C. D. \& Bertozzi, C. R. Synthetic studies toward Mycobacterium tuberculosis sulfolipid-I. J. Org. Chem. 73, 1008-1017 (2008).

77. Crich, D. \& Sun, S. X. Direct chemical synthesis of betamannopyranosides and other glycosides via glycosyl triflates. Tetrahedron 54, 8321-8348 (1998)

78. Crich, D. \& Sun, S. X. Are glycosyl triflates intermediates in the sulfoxide glycosylation method? A chemical and H-1, C-13, and F-19 NMR spectroscopic investigation. J. Am. Chem. Soc. 119, 11217-11223 (1997)

79. Jensen, H. H., Nordstrom, L. U. \& Bols, M. The disarming effect of the 4,6-acetal group on glycoside reactivity: torsional or electronic? J. Am. Chem. Soc. 126, 9205-9213 (2004).

80. Helligso, H. \& Bols, M. Stereoelectronic substituent effects. Acc. Chem. Res. 39, 259-265 (2006). 
81. Zhu, X. M., Kawatkar, S., Rao, Y. \& Boons, G. J. Practical approach for the stereoselective introduction of beta-arabinofuranosides. J. Am. Chem. Soc 128, 11948-11957 (2006).

82. Rademacher, C. et al. Ligand specificity of CS-35, a monoclonal antibody that recognizes mycobacterial lipoarabinomannan: A model system for oligofuranoside - protein recognition. J. Am. Chem. Soc. 129, 10489-10502 (2007).

83. Demchenko, A. V. \& Boons, G. J. A novel direct glycosylation approach for the synthesis of dimers of $\mathrm{N}$-acetylneuraminic acid. Chem. Eur. J. 5, 1278-1283 (1999).

84. De Meo, C. \& Priyadarshani, U. C-5 modifications in N-acetyl-neuraminic acid: scope and limitations. Carbohydr. Res. 343, 1540-1552 (2008).

85. Tanaka, H., Tateno, Y., Nishiura, Y. \& Takahashi, T. Efficient synthesis of an alpha $(2,9)$ trisialic acid by one-pot glycosylation and polymer-assisted deprotection. Org. Lett. 10, 5597-5600 (2008).

86. Crich, D. \& Wu, B. Stereoselective iterative one-pot synthesis of $\mathrm{N}$-glycolylneuraminic acid-containing oligosaccharides. Org. Lett. 10, 4033-4035 (2008)

87. De Meo, C. et al. Solvent effect in the synthesis of sialosyl alpha(2-6) galactosides: is acetonitrile the only choice? Eur. J. Org. Chem. 3673-3677 (2008)

88. Park, J., Kawatkar, S., Kim, J. H. \& Boons, G. J. Stereoselective glycosylations of 2-azido-2-deoxy-glucosides using intermediate sulfonium ions. Org. Lett. 9, 1959-1962 (2007).

89. Benakli, K., Zha, C. X. \& Kerns, R. J. Oxazolidinone protected 2-amino-2-deoxy-D-glucose derivatives as versatile intermediates in stereoselective oligosaccharide synthesis and the formation of alpha-linked glycosides. J. Am. Chem. Soc. 123, 9461-9462 (2001)

90. Manabe, S., Ishii, K. \& Ito, Y. N-benzyl-2,3-oxazolidinone as a glycosyl donor for selective alpha-glycosylation and one-pot oligosaccharide synthesis involving 1,2-cis-glycosylation. J. Am. Chem. Soc. 128, 10666-10667 (2006).

91. Olsson, J. D., Eriksson, L., Lahmann, M. \& Oscarson, S. Investigations of glycosylation reactions with 2-N-acetyl-2N, 3O-oxazolidinone-protected glucosamine donors. J. Org. Chem. 73, 7181-7188 (2008)

92. Manabe, S., Ishii, K., Hashizume, D., Koshino, H. \& Ito, Y. Evidence for endocyclic cleavage of conformationally restricted glycopyranosides. Chem. Eur. J. 15, 6894-6901 (2009).

93. Douglas, N. L., Ley, S. V., Lucking, U. \& Warriner, S. L. Tuning glycoside reactivity: New tool for efficient oligosaccharide synthesis. J. Chem. Soc. Perkin Trans. 1, 51-65 (1998).

94. Zhang, Z. et al. Programmable one-pot oligosaccharide synthesis. J. Am. Chem. Soc. 121, 734-753 (1999).

95. Huang, X. F., Huang, L. J., Wang, H. S. \& Ye, X. S. Iterative one-pot synthesis of oligosaccharides. Angew. Chem. Int. Ed. 43, 5221-5224 (2004).

96. Mong, T. K. K., Lee, H. K., Duron, S. G. \& Wong, C. H. Reactivity-based one-pot total synthesis of fucose GM(1) oligosaccharide: A sialylated antigenic epitope of small-cell lung cancer. Proc. Natl Acad. Sci. USA 100, 797-802 (2003).

97. Wang, Y. H., Huang, X. F., Zhang, L. H. \& Ye, X. S. A four-component one-pot synthesis of alpha-gal pentasaccharide. Org. Lett. 6, 4415-4417 (2004).

98. Tanaka, H., Yamada, H. \& Takahashi, T. Rapid synthesis of oligosaccharides based on one-pot glycosylation. Trends Glycosci. Glycotechnol. 19, 183-193 (2007)

99. Wang, Z., Zhou, L. Y., El-Boubbou, K., Ye, X. S. \& Huang, X. F. Multi-component one-pot synthesis of the tumor-associated carbohydrate antigen Globo-H based on preactivation of thioglycosyl donors. J. Org. Chem. 72, 6409-6420 (2007)
100. Seeberger, P. H. Automated oligosaccharide synthesis. Chem. Soc. Rev 37, 19-28 (2008).

101. Seeberger, P. H. \& Haase, W. C. Solid-phase oligosaccharide synthesis and combinatorial carbohydrate libraries. Chem. Rev. 100, 4349-4394 (2000).

102. Buskas, T., Soderberg, E., Konradsson, P. \& Fraser-Reid, B. Use of $n$-pentenyl glycosides as precursors to various spacer functionalities. J. Org. Chem. 65, 958-963 (2000).

103. Jaipuri, F. A. \& Pohl, N. L. Toward solution-phase automated iterative synthesis: fluorous-tag assisted solution-phase synthesis of linear and branched mannose oligomers. Org. Biomol. Chem. 6, 2686-2691 (2008).

104. Zhang, F., Zhang, W., Zhang, Y., Curran, D. P. \& Liu, G. Synthesis and applications of a light-fluorous glycosyl donor. J. Org. Chem. 74, 2594-2597 (2009).

105. Ko, K. S., Jaipuri, F. A. \& Pohl, N. L. Fluorous-based carbohydrate microarrays. J. Am. Chem. Soc. 127, 13162-13163 (2005).

106. Trincone, A. \& Giordano, A. Glycosyl hydrolases and glycosyltransferases in the synthesis of oligosaccharides. Curr. Org. Chem. 10, 1163-1193 (2006).

107. Lairson, L. L., Henrissat, B., Davies, G. J. \& Withers, S. G. Glycosyltransferases: structures, functions, and mechanisms. Annu. Rev. Biochem. 77, 521-555 (2008).

108. Wong, C. H., Haynie, S. L. \& Whitesides, G. M. Enzyme-catalyzed synthesis of n-acetyllactosamine with in situ regeneration of uridine 5'-diphosphate glucose and uridine 5'-diphosphate galactose. J. Org. Chem. 47, 5416-5418 (1982)

109. Chen, X. et al. Sugar nucleotide regeneration beads (superbeads): a versatile tool for the practical synthesis of oligosaccharides. J. Am. Chem. Soc. 123, 2081-2082 (2001).

110. Leppanen, A. et al. A novel glycosulfopeptide binds to P-selectin and inhibits leukocyte adhesion to P-selectin. J. Biol. Chem. 274, 24838-24848 (1999).

111. Leppanen, A., White, S. P., Helin, J., McEver, R. P. \& Cummings, R. D. Binding of glycosulfopeptides to P-selectin requires stereospecific contributions of individual tyrosine sulfate and sugar residues. Glycobiology 10, 1106-1107 (2000).

112. Rosen, S. D. \& Bertozzi, C. R. The selectins and their ligands. Curr. Opin. Cell Biol. 6, 663-673 (1994).

113. Naruchi, K. et al. Construction and structural characterization of versatile lactosaminoglycan-related compound library for the synthesis of complex glycopeptides and glycosphingolipids. J. Org. Chem. 71, 9609-9621 (2006).

114. Yu, H. \& Chen, X. Carbohydrate post-glycosylational modifications. Org. Biomol. Chem. 5, 865-872 (2007).

115. Chokhawala, H. A. et al. Combinatorial chemoenzymatic synthesis and highthroughput screening of sialosides. ACS Chem. Biol. 3, 567-576 (2008).

116. Hancock, S. M., Vaughan, M. D. \& Withers, S. G. Engineering of glycosidases and glycosyltransferases. Curr. Opin. Chem. Biol. 10, 509-519 (2006).

117. Jahn, M. et al. Expansion of the glycosynthase repertoire to produce defined manno-oligosaccharides. Chem. Commun. 1327-1329 (2003).

118. Li, B., Zeng, Y., Hauser, S., Song, H. J. \& Wang, L. X. Highly efficient endoglycosidase-catalyzed synthesis of glycopeptides using oligosaccharide oxazolines as donor substrates. J. Am. Chem. Soc. 127, 9692-9693 (2005).

119. Umekawa, M. et al. Mutants of Mucor hiemalis endo-beta-Nacetylglucosaminidase show enhanced transglycosylation and glycosynthaselike activities. J. Biol. Chem. 283, 4469-4479 (2008).

\section{Acknowledgements}

This research was supported by the National Institute of General Medicine (NIGMS) of the National Institutes of Health (Grant No 2R01GM065248) and the National Cancer Institute of the National Institutes of Health (Grant No. R01CA088986). 